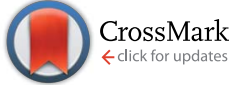

Cite this: RSC Adv., 2017, 7, 6875

Received 29th November 2016 Accepted 4th January 2017

DOI: 10.1039/c6ra27499d

www.rsc.org/advances

\title{
Tuning strain and photoluminescence of confined Au nanoparticles by hydrogen passivation
}

\author{
Cailei Yuan, ${ }^{* a}$ Yaxing Mei, ${ }^{a}$ Ting $Y u,{ }^{a}$ Yong Yang, ${ }^{a}$ Qinliang li, ${ }^{a}$ Aijun Hong, ${ }^{a}$ Keng $\mathrm{Xu}{ }^{a}$ \\ Xingfang Luo, *a Jun $\mathrm{He}^{\mathrm{b}}$ and Wen Lei ${ }^{\mathrm{c}}$
}

\begin{abstract}
$\mathrm{Au}$ nanoparticles confined in an $\mathrm{Al}_{2} \mathrm{O}_{3}$ matrix are synthesized using pulsed laser deposition method and rapid thermal annealing technique. The confined $\mathrm{Au}$ nanoparticles experience a compressive strain during the growth process. It is demonstrated that hydrogen passivation can be used to enhance and tailor the optical properties of confined Au nanoparticles by engineering the strain and defect states of the confined Au nanoparticles. The findings provide an insight and useful methodology to improve the emission efficiency of noble metal nanoparticle based materials for potential application in optoelectronic and photonic devices.
\end{abstract}

\section{Introduction}

Recently, the optical properties of noble metal nanoparticles, particularly surface plasmon resonance in the visible region, have attracted significant attention due to the potential applications in optoelectronic and photonic devices..$^{1-5}$ Among those noble metal nanoparticles, $\mathrm{Au}$ nanoparticles are promising materials for fabricating light emitting devices due to their strong surface plasmonic effect. ${ }^{6,7}$ As a result, photoluminescence (PL) of Au nanoparticles has become a focus of study. ${ }^{8,9}$ To achieve their final device applications, substantial study has been carried out to understand the light emission process and improve the emission efficiency of $\mathrm{Au}$ nanoparticles. For practical device applications, Au nanoparticles are usually embedded in a solid matrix. However, because of the thermal expansion mismatch between Au nanoparticles and the surrounding matrix, substantial strain is generated and accumulated inside and around Au nanoparticles during the growth process. ${ }^{10,11}$ The accumulation and relaxation of strain can lead to the generation of defects in the nanoparticles and in the vicinity of the interface between the nanoparticles and the surrounding matrix. ${ }^{11,12}$ These bulk and interfacial defects can degrade the physical properties of Au nanoparticles such as carrier lifetime, which can have a disastrous impact on the final device performance. ${ }^{13}$ Because of the large surface/volume ratio of nanoparticles, the impact of interfacial defects on the optical

ajiangxi Key Laboratory of Nanomaterials and Sensors, School of Physics, Communication and Electronics, Jiangxi Normal University, 99 Ziyang Avenue, Nanchang 330022, Jiangxi, China.E-mail: clyuan@jxnu.edu.cn; xfluo@jxnu.edu.cn

${ }^{b}$ Hunan Key Laboratory for Super-micro Structure and Ultrafast Process, School of Physics and Electronics, Central South University, 932 South Lushan Road, Changsha 410083, Hunan, China

${ }^{c}$ School of Electrical, Electronic and Computer Engineering, The University of Western Australia, 35 Stirling Highway, Crawley 6009, Australia properties of $\mathrm{Au}$ nanoparticles can be even more significant. Thus, to achieve high performance functioning devices based on confined Au nanoparticles, bulk and interfacial defects as well as their related defect energy states must be eliminated or inactivated.

Passivation provides an effective methodology to eliminate the defect energy states or make them inactive. $\mathrm{SiO}_{2}$ and $\mathrm{Si}_{3} \mathrm{~N}_{4}$ surface passivation has been widely applied to passivate $\mathrm{Si}$ solar cells to achieve high device performance via bonding the dangling bonds at the Si semiconductor surfaces. ${ }^{14,15}$ Apart from surface passivation, bulk passivation can also help to eliminate the defect energy states in materials. For example, hydrogen atoms have been used to passivate the defects in bulk $\mathrm{HgCdTe}$ infrared materials. ${ }^{16}$ Hydrogen atoms have also been used to passivate Si nanoparticles, leading to a significant enhancement of the PL emission efficiency by passivating the nonradiative defects at the interface between Si nanoparticles and the surrounding matrix. ${ }^{17,18}$ However, little attention has been devoted to enhancing the light emission efficiency of $\mathrm{Au}$ nanoparticles. Herein, we present a study on hydrogen passivation of $\mathrm{Au}$ nanoparticles embedded in an $\mathrm{Al}_{2} \mathrm{O}_{3}$ matrix. After hydrogen passivation, the PL emission intensity increased three-fold, which indicates that hydrogen passivation can effectively eliminate or inactivate the defect states in $\mathrm{Au}$ nanoparticles.

\section{Experimental}

$\mathrm{Au}$ nanoparticles confined in an amorphous $\mathrm{Al}_{2} \mathrm{O}_{3}$ matrix were synthesized using pulsed laser deposition (PLD) method and rapid thermal annealing (RTA) technique. Briefly, a KrF pulsed laser beam with a $248 \mathrm{~nm}$ wavelength and a frequency of $10 \mathrm{~Hz}$ was used to ablate the target. A part of high-purity (99.99\%) circular $\mathrm{Al}_{2} \mathrm{O}_{3}$ plate (about $40 \mathrm{~mm}$ in diameter) and a part of 
high-purity (99.99\%) Au square plate (about $8 \mathrm{~mm}$ in length) were used as the targets. The deposition of the film on the $\mathrm{Si}$ substrate was carried out inside a high-vacuum chamber at a background pressure of about $1 \times 10^{-7}$ Torr, with the substrate kept at room temperature. After the deposition, the sample was subjected to RTA at $800{ }^{\circ} \mathrm{C}$ for $60 \mathrm{~s}$ under either pure $\mathrm{N}_{2}$ or $10 \% \mathrm{H}_{2} / \mathrm{Ar}$ atmosphere, and the nanoparticles obtained were labeled as nitrogen-annealed or hydrogen-annealed $\mathrm{Au}$ nanoparticles. The structure of the deposited film was examined via high resolution transmission electron microscopy (HRTEM) with a JEOL 2010 microscope. The TEM electron diffraction pattern was matched against a simulated diffraction pattern generated using the Java Electron Microscopy Simulation (JEMS) software. ${ }^{\mathbf{1 0 , 1 1}} \mathrm{X}$-ray photoelectron spectroscopy (XPS) measurements were carried out with a Kratos XSAM800 spectrometer using $\mathrm{Al} \mathrm{K} \alpha$ radiation. $\mathrm{PL}$ and Raman spectroscopy measurements were carried out at room temperature with a HORIBA LabRAM HR Evolution instrument, at an excitation wavelength of $325 \mathrm{~nm}$ and $514 \mathrm{~nm}$, respectively.

\section{Results and discussion}

Fig. 1(a) shows the HRTEM image of the hydrogen-annealed Au nanoparticles confined in the $\mathrm{Al}_{2} \mathrm{O}_{3}$ matrix. It can be seen that a large number of $\mathrm{Au}$ nanoparticles are formed in the $\mathrm{Al}_{2} \mathrm{O}_{3}$ matrix. Fig. 1(b) displays the HRTEM image of a single hydrogen-annealed $\mathrm{Au}$ nanoparticle confined in the $\mathrm{Al}_{2} \mathrm{O}_{3}$ matrix. Clearly, the $\mathrm{Al}_{2} \mathrm{O}_{3}$ matrix still remains amorphous after annealing under hydrogen atmosphere, whereas the Au nanoparticles are crystalline with a spherical shape. The average size of the hydrogen-annealed $\mathrm{Au}$ nanoparticles is about $5 \mathrm{~nm}$. Fig. 1(c) shows the electron diffraction pattern of the hydrogenannealed $\mathrm{Au}$ nanoparticles. The diffraction pattern was matched against a simulated diffraction pattern generated via the JEMS software. By comparing the experimental diffraction

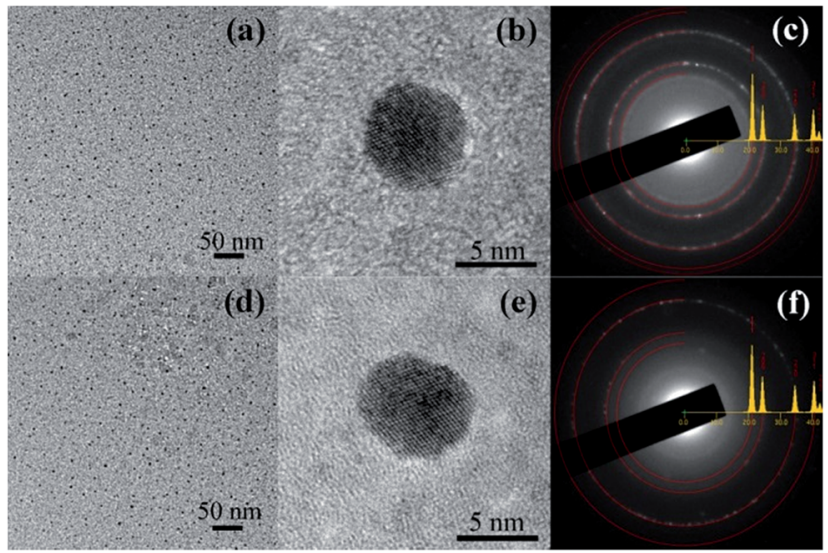

Fig. 1 (a) HRTEM image of hydrogen-annealed $A u$ nanoparticles confined in the $\mathrm{Al}_{2} \mathrm{O}_{3}$ matrix; (b) HRTEM image of a single hydrogenannealed Au nanoparticle; (c) electron diffraction pattern of hydrogenannealed Au nanoparticles; (d) HRTEM image of nitrogen-annealed Au nanoparticles confined in the $\mathrm{Al}_{2} \mathrm{O}_{3}$ matrix; (e) HRTEM image of a single nitrogen-annealed $\mathrm{Au}$ nanoparticle; (f) electron diffraction pattern of nitrogen-annealed Au nanoparticles. pattern with the simulated one, it could be concluded that the hydrogen-annealed Au nanoparticles have a cubic crystal structure (space group Fm $\overline{3} \mathrm{~m}$ ). Fig. 1(d) shows the HRTEM image of a single nitrogen-annealed $\mathrm{Au}$ nanoparticle confined in the $\mathrm{Al}_{2} \mathrm{O}_{3}$ matrix. Similarly, a large number of $\mathrm{Au}$ nanoparticles are formed in the $\mathrm{Al}_{2} \mathrm{O}_{3}$ matrix, and the $\mathrm{Al}_{2} \mathrm{O}_{3}$ matrix remains amorphous after annealing under nitrogen atmosphere. The average size of the nitrogen-annealed Au nanoparticles was also around $5 \mathrm{~nm}$. Fig. 1(e) presents the HRTEM image of a single nitrogen-annealed $\mathrm{Au}$ nanoparticle confined inside the $\mathrm{Al}_{2} \mathrm{O}_{3}$ matrix. It can be seen that crystalline $\mathrm{Au}$ nanoparticles are formed in the $\mathrm{Al}_{2} \mathrm{O}_{3}$ matrix. Fig. 1(f) shows the electron diffraction pattern of the nitrogen-annealed $\mathrm{Au}$ nanoparticles. Similarly, by comparing the experimental diffraction pattern with the simulated one, it could be concluded that nitrogen-annealed Au nanoparticles also have a cubic crystal structure (space group $\mathrm{Fm} \overline{3} \mathrm{~m}$ ). Therefore, annealing under different atmospheres does not have much of an effect on the crystal structure, size and morphology of the Au nanoparticles confined in the $\mathrm{Al}_{2} \mathrm{O}_{3}$ matrix.

To confirm the chemical composition of the $\mathrm{Au}$ nanoparticles confined in the $\mathrm{Al}_{2} \mathrm{O}_{3}$ matrix, XPS measurements were performed on the Au nanoparticles annealed as well as on pure bulk $\mathrm{Au}$, for reference. Fig. 2 shows the high-resolution XPS spectra of the $\mathrm{Au}$ (4f) peaks for bulk $\mathrm{Au}$, hydrogen-annealed $\mathrm{Au}$ and nitrogen-annealed $\mathrm{Au}$ nanoparticles confined in the $\mathrm{Al}_{2} \mathrm{O}_{3}$ matrix. The doublet peaks of $\mathrm{Au}\left(4 \mathrm{f}_{7 / 2}\right)$ and $\mathrm{Au}\left(4 \mathrm{f}_{5 / 2}\right)$, located at 84.1 and $87.8 \mathrm{eV}$, respectively, can be clearly observed for bulk $\mathrm{Au}$, which can be attributed to the presence of $\mathrm{Au}$ in the metallic state $\left(\mathrm{Au}^{0}\right) .{ }^{19}$ However, four peaks can be clearly observed in the XPS spectrum of the nitrogen-annealed $\mathrm{Au}$ nanoparticles. The doublet peaks at 84.1 and $87.8 \mathrm{eV}$ are characteristic for metallic $\mathrm{Au}$. The two very small peaks at 84.9 and $88.6 \mathrm{eV}$ can be assigned to the ionic gold species $\mathrm{Au}^{\delta+}$, which is in line with data reported in literature, ${ }^{20}$ indicating the existence of bulk and interfacial defects in $\mathrm{Au}$ nanoparticles. In contrast, for the hydrogenannealed Au nanoparticles, only the doublet peaks at 84.1 and

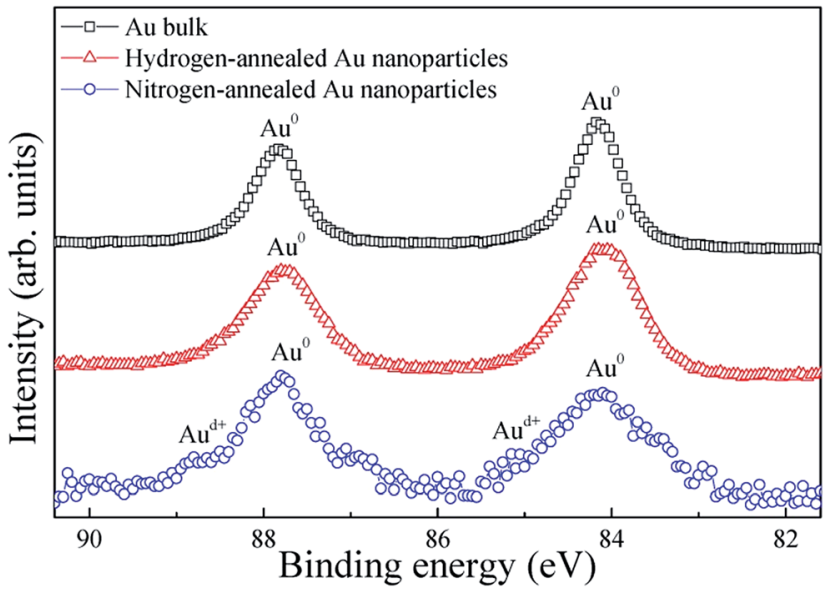

Fig. 2 XPS spectra of Au $4 f$ electrons from Au bulk, hydrogenannealed and nitrogen-annealed $\mathrm{Au}$ nanoparticles confined in the $\mathrm{Al}_{2} \mathrm{O}_{3}$ matrix. 
$87.8 \mathrm{eV}$ can be observed, which indicates that $\mathrm{Au}$ nanoparticles remain in the metallic crystalline phase after annealing. This result clearly demonstrates that hydrogen atoms can bond with the dangling bonds, and thus passivate interfacial defects on the surface of the Au nanoparticles, as well as the defects inside the nanoparticles.

Because of the thermal expansion mismatch between $\mathrm{Au}$ nanoparticles and $\mathrm{Al}_{2} \mathrm{O}_{3}$, the formation and growth of $\mathrm{Au}$ nanoparticles in the $\mathrm{Al}_{2} \mathrm{O}_{3}$ matrix via $\mathrm{PLD}$ and RTA are accompanied by the generation and accumulation of strain. During the growth process, Au nanoparticles will suffer a substantial compressive strain due to volume expansion whilst confined within the $\mathrm{Al}_{2} \mathrm{O}_{3}$ matrix. However, strain can have a significant impact on the generation and distribution of defects, and subsequently on the optical properties of $\mathrm{Au}$ nanoparticles. Thus, it is essential to have an understanding about the strain and its distribution in Au nanoparticles as well as in the surrounding matrix. In order to understand the strain within the system, the strain distribution in Au nanoparticles confined in the $\mathrm{Al}_{2} \mathrm{O}_{3}$ matrix was qualitatively simulated using finite element (FE) calculations, which are performed with the commercial ANSYS software package. ${ }^{\mathbf{1 0 , 1 1}}$ The Young's modulus is taken to be 170 and $360 \mathrm{GPa}$ for $\mathrm{Au}$ and $\mathrm{Al}_{2} \mathrm{O}_{3}$, respectively, while the Poisson's ratio is taken to be 0.42 and 0.24 for $\mathrm{Au}$ and $\mathrm{Al}_{2} \mathrm{O}_{3}$, respectively. Fig. 3(a) shows the cross-sectional strain distribution for a $\mathrm{Au}$ nanoparticle grown in an $\mathrm{Al}_{2} \mathrm{O}_{3}$ matrix. Correspondingly, Fig. 3(b) shows the $X-Y$ plane strain profile for a $\mathrm{Au}$ nanoparticle grown in an $\mathrm{Al}_{2} \mathrm{O}_{3}$ matrix. As expected, the $\mathrm{Au}$ nanoparticle suffers compressive strain from the $\mathrm{Al}_{2} \mathrm{O}_{3}$ matrix. Moreover, an inhomogeneous strain distribution can be observed, which is denoted by the different colors as labeled by the marks in Fig. 3(a). It can also be observed that the strain at the center of the Au nanoparticle is distributed homogeneously and the strain at the surface of the nanoparticle is weaker than that at the center of the nanoparticles. As a matter of fact, because of the large surface/volume ratio in nanoparticles, the inhomogeneous strain distribution can have a significant impact on interfacial defect states, and consequently on the optical properties of isolated nanoparticles, which will be discussed later.

To further study the strain in Au nanoparticles, Raman spectroscopy measurements were performed on the annealed
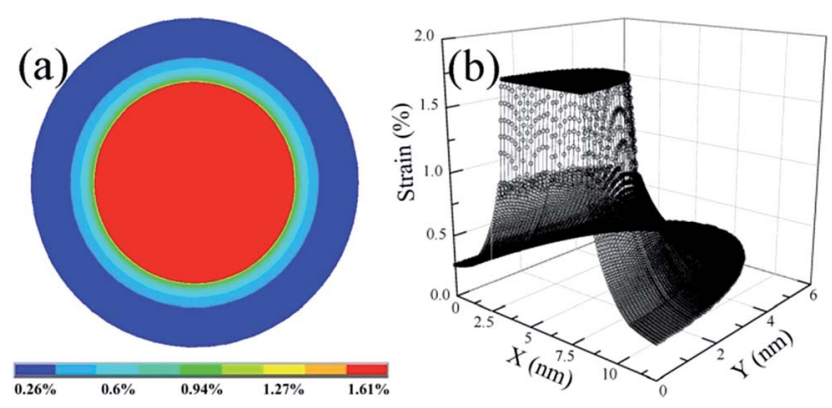

Fig. 3 (a) Cross-sectional strain distribution in a $\mathrm{Au}$ nanoparticle confined in an $\mathrm{Al}_{2} \mathrm{O}_{3}$ matrix; (b) $X-Y$ plane strain profile of $\mathrm{Au}$ nanoparticles confined in an $\mathrm{Al}_{2} \mathrm{O}_{3}$ matrix.

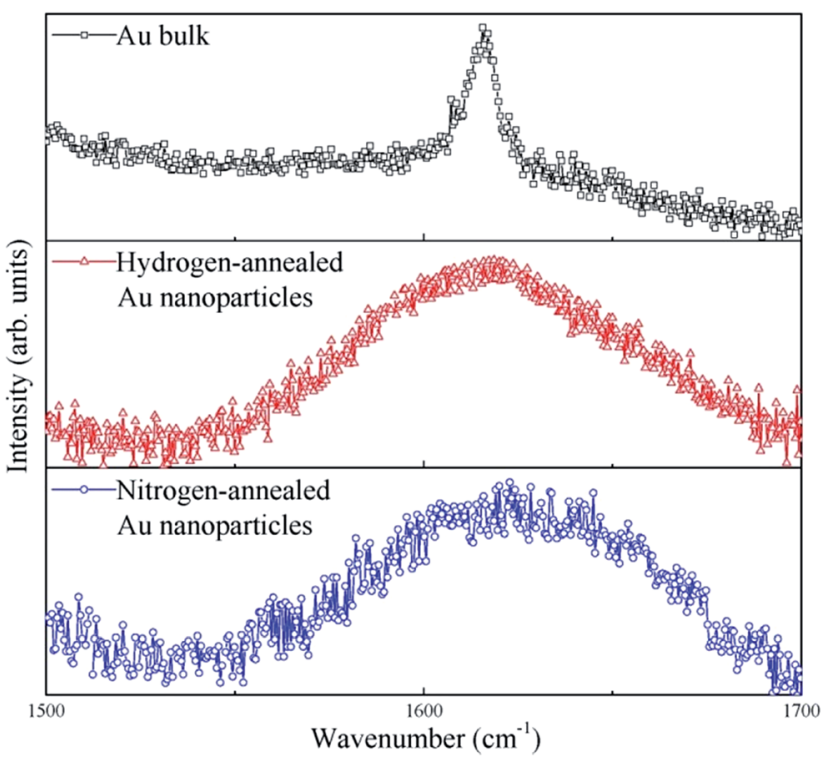

Fig. 4 Raman spectra of bulk Au, hydrogen-annealed and nitrogenannealed Au nanoparticles excited at a wavelength of $518 \mathrm{~nm}$.

$\mathrm{Au}$ nanoparticles as well as on bulk $\mathrm{Au}$, for reference. Fig. 4 displays the room temperature Raman spectra of bulk, hydrogen-annealed and nitrogen-annealed $\mathrm{Au}$ nanoparticles excited at a wavelength of $518 \mathrm{~nm}$. A Raman peak centered at about $1615 \mathrm{~cm}^{-1}$ can be clearly observed for bulk Au. ${ }^{21}$ On the other hand, a Raman peak centered at about $1618 \mathrm{~cm}^{-1}$ and $1623 \mathrm{~cm}^{-1}$ can be observed for hydrogen-annealed and nitrogen-annealed $\mathrm{Au}$ nanoparticles, respectively. The Raman peak of $\mathrm{Au}$ nanoparticles confined in $\mathrm{Al}_{2} \mathrm{O}_{3}$ matrix displays a blue-shift. This clearly demonstrates that $\mathrm{Au}$ nanoparticles grown inside an $\mathrm{Al}_{2} \mathrm{O}_{3}$ matrix are under significant compressive strain, ${ }^{22,23}$ which is consistent with the strain simulation results. Moreover, it should also be noted that the peak shift of the nitrogen-annealed $\mathrm{Au}$ nanoparticles is larger than that of the hydrogen-annealed Au nanoparticles, which also indicates that the compressive strain in nitrogen-annealed Au nanoparticles is larger than that in the hydrogen-annealed $\mathrm{Au}$ nanoparticles. This might be due to the fact that hydrogen atoms can bond with the dangling bonds, and thus passivate interfacial defects on the surface and inside the $\mathrm{Au}$ nanoparticles, leading to a relaxation in the network strain. Hydrogen atoms have a smaller volume and higher reactivity than nitrogen atoms; this makes it easier for hydrogen to diffuse into Au nanoparticles and passivate the defects, leading to a dramatic impact on the optical properties of Au nanoparticles.

Fig. 5 shows the PL spectra recorded at room temperature for amorphous $\mathrm{Al}_{2} \mathrm{O}_{3}$ thin films, hydrogen-annealed and nitrogenannealed $\mathrm{Au}$ nanoparticles confined in an amorphous $\mathrm{Al}_{2} \mathrm{O}_{3}$ matrix. Clearly, no PL peaks can be observed for the reference amorphous $\mathrm{Al}_{2} \mathrm{O}_{3}$ thin film. In contrast, a PL band centered at around $585 \mathrm{~nm}$ can be clearly observed for samples having $\mathrm{Au}$ nanoparticles confined in the amorphous $\mathrm{Al}_{2} \mathrm{O}_{3}$ matrix. Therefore, the observed PL peak can be attributed to the Au nanoparticles. As can be seen in Fig. 5, the PL peak intensity of the Au 


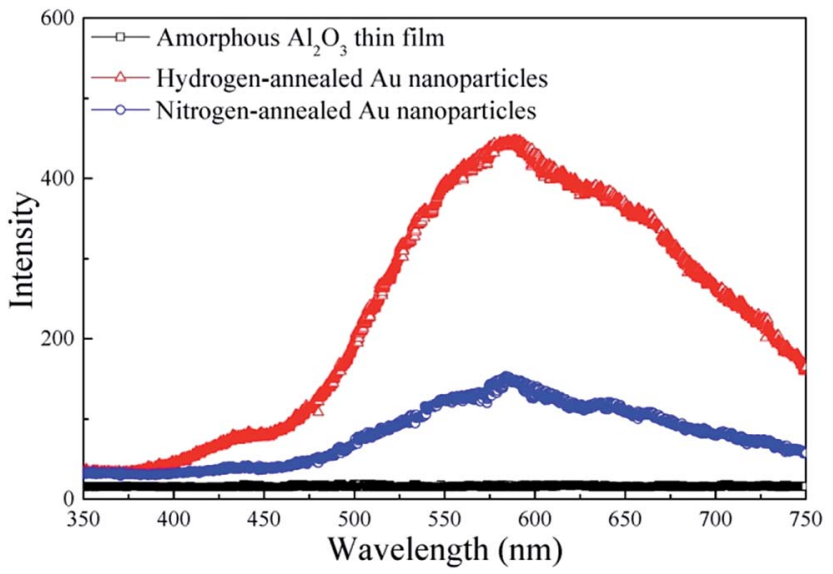

Fig. $5 \mathrm{PL}$ spectra of amorphous $\mathrm{Al}_{2} \mathrm{O}_{3}$ thin films, hydrogen-annealed Au nanoparticles and nitrogen-annealed Au nanoparticles.

nanoparticles annealed under hydrogen atmosphere is around three times that of $\mathrm{Au}$ nanoparticles annealed under nitrogen atmosphere. This is mainly due to the fact that defects in the $\mathrm{Au}$ nanoparticles and in the vicinity of Au nanoparticles are effectively passivated by hydrogen atoms, leading to an enhanced light emission efficiency. As discussed previously, when spherical $\mathrm{Au}$ nanoparticles are formed in the $\mathrm{Al}_{2} \mathrm{O}_{3}$ matrix, the matrix exerts an isotropic pressure on the nanoparticles, giving rise to a compressive strain in the Au nanoparticles. The strain and its relaxation could result in several defect states inside the Au nanoparticle and in the vicinity of the interface between $\mathrm{Au}$ nanoparticles and $\mathrm{Al}_{2} \mathrm{O}_{3}$ matrix, ultimately also resulting in several nonradiative surface defects, which can causes low PL emission efficiency for $\mathrm{Au}$ nanoparticles. ${ }^{24}$ As indicated from the Raman spectra, the compressive strain in the hydrogenannealed $\mathrm{Au}$ nanoparticles was less than that in the nitrogenannealed $\mathrm{Au}$ nanoparticles. Therefore, hydrogen-annealed Au nanoparticles had less defect states. More importantly, annealing under hydrogen atmosphere also allows the hydrogen atoms to passivate the defect states and eliminate the non-radiative defects in the $\mathrm{Au}$ nanoparticles, ${ }^{17}$ which is consistent with results of the XPS spectra. As a result, the PL emission intensity of hydrogen-annealed $\mathrm{Au}$ nanoparticles is effectively enhanced, being almost 3 -fold that of nitrogenannealed Au nanoparticles.

\section{Conclusion}

In summary, $\mathrm{Au}$ nanoparticles confined in an $\mathrm{Al}_{2} \mathrm{O}_{3}$ matrix are synthesized using the PLD method and RTA technique. The confined Au nanoparticles experience a compressive strain during the growth process. It is found that annealing under hydrogen atmosphere can relax compressive strain in the $\mathrm{Au}$ nanoparticles, and effectively passivate their defect states, leading to an enhanced emission efficiency. The findings presented here provide an insight and offer a useful methodology to enhance the emission efficiency of noble metal nanoparticlebased materials for potential applications in optoelectronic and photonic devices.

\section{Acknowledgements}

This study was supported by the Natural Science Foundation of China (Grant No. 51461019, 51661012, 51361013, 51561012 and 61664005), the Project for Young Scientist Training of Jiangxi Province (Grant No. 20153BCB23016), the Natural Science Foundation of Jiangxi Province (Grant No. 20151BAB202004 and 20161BAB201022), and the Australian Research Council (Grant No. FT130101708, DP170104562 and LE170100233).

\section{References}

1 H. M. Hiep, H. Yoshikawa, M. Saito and E. Tamiya, ACS Nano, 2009, 3, 446.

2 S. Murai, Y. Fujimoto, K. Iwata, K. Fujita and K. Tanaka, Appl. Phys. Lett., 2011, 98, 121917.

3 K. M. Guo, M. Y. Li, X. L. Fang, X. L. Liu, B. Sebo, Y. D. Zhu, Z. Q. Hu and X. Z. Zhao, J. Power Sources, 2013, 230, 155.

4 Z. B. Zhan, R. Xu, X. Z. Zheng, Q. Fu, M. H. Wu and Y. Lei, Nanotechnology, 2016, 27, 445301.

5 V. Garg, B. S. Sengar, V. Awasthi, Aaryashree, P. Sharma, C. Mukherjee, S. Kumar and S. Mukherjee, RSC Adv., 2016, 6, 26216.

6 Y. H. Lee, D. H. Kim, K. H. Yoo and T. W. Kim, Appl. Phys. Lett., 2014, 105, 183303.

7 D. H. Kim and T. W. Kim, Org. Electron., 2016, 34, 270.

8 Y. Ishida, T. Sumi and T. Yonezawa, New J. Chem., 2015, 39, 5895.

9 J. Prakash, V. Kumar, R. E. Kroon, K. Asokan, V. Rigato, K. H. Chae, S. Gautam and H. C. Swart, Phys. Chem. Chem. Phys., 2016, 18, 2468.

10 C. L. Yuan, Z. X. Jiang and S. L. Ye, Nanoscale, 2014, 6, 1119. 11 C. L. Yuan, Y. Zhang, J. He, S. L. Ye, W. Lei, X. S. Liu and G. Gu, J. Mater. Chem. C, 2014, 2, 8768.

12 Z. X. Jiang, C. L. Yuan and S. L. Ye, RSC Adv., 2014, 4, 19584. 13 J. Xu, J. Moxom, B. Somieski, C. W. White, A. P. Mills, R. Suzuki and S. Ishibashi, Phys. Rev. B: Condens. Matter Mater. Phys., 2001, 64, 113404.

14 K. Watanabe, R. Tsuchiya, T. Mine, Y. Yonamoto, N. Akamatsu and M. Hatano, Appl. Phys. Lett., 2012, 101, 153902.

15 C. A. Lin, K. Y. Lai, W. C. Lien and J. H. He, Nanoscale, 2012, 4, 6520.

16 W. D. Hu, X. S. Chen, Z. H. Ye and W. Lu, Appl. Phys. Lett., 2011, 99, 091101.

17 S. Cheylan and R. G. Elliman, Appl. Phys. Lett., 2001, 78, 1225. 18 S. P. Withrow, C. W. White, A. Meldrum, J. D. Budai Jr, D. M. Hembree and J. C. Barbour, J. Appl. Phys., 1999, 86, 396.

19 J. H. Liu, G. Liu, C. Liu, W. X. Li and F. Wang, Catal. Sci. Technol., 2016, 6, 2055.

20 D. A. Bulushev, I. Yuranov, E. I. Suvorova, P. A. Buffat and L. Kiwi-Minsker, J. Catal., 2004, 224, 8.

21 Q. Fu, K. M. Wong, Y. Zhou, M. H. Wu and Y. Lei, RSC Adv., 2015, 5, 6172.

22 M. Montazeri, M. Fickenscher, L. M. Smith, H. E. Jackson, J. Yarrison-Rice, J. H. Kang, Q. Gao, H. H. Tan, C. Jagadish, 
Y. N. Guo, J. Zou, M. E. Pistol and C. E. Pryor, Nano Lett., 2010, 10, 880.

23 Q. Wang, Y. X. Gao, G. M. Mao, H. Liu and X. M. Ren, Appl. Phys. Lett., 2015, 107, 082108.
24 L. Ravelli, C. Macchi, S. Mariazzi, P. Mazzoldi, W. Egger, C. Hugenschmidt, A. Somoza and R. S. Brusa, J. Phys. D: Appl. Phys., 2015, 48, 495302. 